\title{
Kinetics towards mechanism and real operation for ultra-deep hydrodesulfurization and hydrodenitrogenation of diesel
}

\author{
Yachen $\mathrm{Yin}^{1}$, Wenbin $\mathrm{Chen}^{2}$, Guilian $\mathrm{Wu}^{1}$, Feng Xin ${ }^{1}$, Kang Qin ${ }^{2}$, Yutao $\mathrm{Lu}^{2}$, Le Zhang ${ }^{2}$, \\ and Mingfeng $\mathrm{Li}^{2}$ \\ ${ }^{1}$ Tianjin University \\ ${ }^{2}$ Research Institute of Petroleum Processing, SINOPEC, 18 Xue Yuan Road
}

May 6, 2020

\begin{abstract}
As the hydrodesulfurization (HDS) of diesel achieves ultra-deepness, our understanding of its kinetics is still far from in-depth. Therefore, herein, two lumped kinetic models for the ultra-deep hydrodesulfurization (UHDS) and hydrodenitrogenation (HDN) are established based on experiments under a wide range of operating conditions. Meanwhile, a four-lump kinetic model of the aromatic hydrosaturation (AHS) is erected. Our kinetic models disclose thermodynamic decisiveness in UHDS, which is unreachable beyond a temperature upper limit or a pressure lower limit. We also reveals the unexpected temperature dependence of nitrogen inhibition to HDS, for less than 300 the nitrogen inhibition becomes even more potent despite nitrogen removal by HDN reactions. Subsequently, the HDS kinetics of total sulfur are deciphered as multi stages exist in the whole reaction coordinate. Accordingly, a four-stage conceptual model involving mechanism and rate laws is proposed to offer a better understanding of nitrogen inhibition, thermodynamics and kinetics in UHDS.
\end{abstract}

\section{Hosted file}

Kinetics towards mechanism and real operation for ultra-deep hydrodesulfurization and hydrodenitrogen.p available at https://authorea.com/users/317248/articles/447384-kinetics-towards-mechanismand-real-operation-for-ultra-deep-hydrodesulfurization-and-hydrodenitrogenation-ofdiesel 